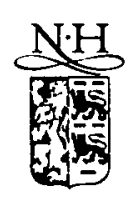

\title{
Sum-accelerated pseudospectral methods: finite differences and sech-weighted differences
}

\author{
John P. Boyd \\ Department of Atmospheric, Oceanic \& Space Sciences, University of Michigan, 2455 Hayward Avenue, \\ Ann Arbor, MI 48109, USA
}

\begin{abstract}
This work continues our previous studies of algorithms for accelerating the convergence of pseudospectral derivative series in order to obtain new differentiation schemes which have the sparsity and low cost of finite differences but the accuracy of spectral methods. We develop a general theoretical framework for difference schemes. Finite differences are close to optimum, but can be bettered by a new scheme we have dubbed 'sech-weighted' differences. Through numerical examples, we show that sech-weighted differences are effective. In contrast, non-linear accelerations like Padé approximants and the Levin $u$-transform, so popular in other applications, are inferior for approximating derivatives to linear accelerations like finite differences, sech-weighted differences and the Euler method.
\end{abstract}

\section{Introduction}

The goal of this work is to hybridize pseudospectral and finite difference algorithms in order to combine the best advantages of both. In earlier articles $[1,2]$, we have shown how pseudospectral derivative series can be accelerated to produce a difference scheme which is spectrally accurate, but has the low cost and matrix bandwidth of a finite difference method. In this paper, we describe a couple of additional variations on this theme.

For simplicity, we consider an evenly spaced infinite grid and assume that our solution $u(x)$ decays exponentially as $|x| \rightarrow \infty$. This eliminates boundary effects. For the infinite interval, a good basis is

$$
\begin{aligned}
& C_{j}(x ; h) \equiv \operatorname{sinc}\left(\left[x-x_{j}\right] / h\right), \\
& x_{j} \equiv j h, \quad j=0, \pm 1, \pm 2, \pm \cdots, \\
& \operatorname{sinc}(x) \equiv \sin (\pi x) /(\pi x) .
\end{aligned}
$$

Sinc methods are described in [1,3] and especially [4]. As clearly illustrated in [2], however, sum acceleration ideas apply equally well to Chebyshev and Fourier pseudospectral methods.

If we define the scaled differential operator

$$
D_{m} \equiv i^{-m} h^{m} \frac{\mathrm{d}^{m}}{\mathrm{~d} x^{m}}
$$

and a centered difference approximation to $D_{m}$ by

$$
D_{m}^{\mathrm{app}} u(x) \equiv \sum_{j=-n}^{n} \delta_{j}^{(m)} u(x+j h),
$$


and introduce the scaled wavenumber

$$
K \equiv k h \text {, }
$$

so that

$$
D_{m} \mathrm{e}^{\mathrm{i} k x} \equiv K^{m} \mathrm{e}^{\mathrm{i} k x}
$$

then

$$
\begin{aligned}
& D_{2 m+1}^{\mathrm{app}} \mathrm{e}^{\mathrm{i} k x} \equiv\left\{2 \sum_{j=1}^{n} \delta_{j}^{(2 m+1)} \sin (j K)\right\} \mathrm{e}^{\mathrm{i} k x}, \\
& D_{2 m}^{\mathrm{app}} \mathrm{e}^{\mathrm{i} k x} \equiv\left\{\delta_{0}^{(2 m)}+2 \sum_{j=1}^{n} \delta_{j}^{(2 m)} \cos (j K)\right\} \mathrm{e}^{\mathrm{i} k x} .
\end{aligned}
$$

Here, we have used the fact that $\exp (\mathrm{i} k x)$ is an eigenfunction of the translation operator $E$ with eigenfunction $\exp (\mathrm{i} k h)$ where the translation operator is defined by $E u(x) \equiv u(x+h)$.

The set $\{\exp (\mathrm{i} k x), k$ real $\}$ is a complete basis for the infinite interval. Therefore, the difference formulas will accurately approximate the derivatives of a function only to the extent that the sums in braces in Eqs. (1.8) and (1.9) approximate $K^{m}$ for all $K$.

One species of error is unavoidable, independent of the difference weights $\delta_{j}^{m}$. Because the Fourier series in (1.8) and (1.9) are periodic with period $2 \pi$ in $K$, whereas the powers of $K$ are non-periodic and unbounded, it follows that a wavenumber $K$ such that $|K|>\pi$ will be differentiated as if it were the wavenumber $K^{\prime}=K-2 \pi p$ where $p$ is an integer such that $\left|K^{\prime}\right| \leqslant \pi$. This apparent shift of wavenumber is called 'aliasing', so we will refer to this error as the aliasing error.

There is no way to control or reduce aliasing error except by decreasing the grid spacing $h$. All wavenumbers above the 'aliasing limit'

$$
K_{\text {limit }} \equiv \pi \Leftrightarrow k_{\text {limit }} \equiv \pi / h
$$

are still aliased, but decreasing $h$ increases $k_{\text {limit }}$. If $u(x)$ is analytic for real $x$ and decays exponentially as $|x| \rightarrow \infty$, then its Fourier transform will decay exponentially for $|k| \gg 1$. This in turn implies an exponentially fast decrease of aliasing error with $1 / h$.

Our focus is the second source of error: how accurately the truncated Fourier series approximate the powers of $K$ for wavenumbers within the aliasing limit. This error is controlled by the difference weights.

In the next two sections, we discuss two alternatives for choosing the weights. The first is to use the Fourier series for the powers of $K^{m}$. This gives both the standard sinc pseudospectral method (in the limit $n \rightarrow \infty$ ) and the 'truncated sinc' method (for finite $n$ ). The second option is finite differences, which is equivalent to applying a certain sum acceleration method to the classical sinc pseudospectral algorithm.

In Section 4, we explain why neither of these two schemes is entirely satisfactory. We prove an elementary theorem which specifies the unique set of expansion weights which is optimum for a given function $u(x)$. In brief, the answer is to make (1.8) and (1.9) the weighted least squares expansions of $K^{m}$ with a weight function equal to the square of $U(k)$, the Fourier transform of $u(x)$.

As it stands, this prescription is useless because we do not know $U(k)$ in advance; if we did, we would not need a pseudospectral method! However, the general form of $U(k)$ for a broad class of functions suggests the weight function $\operatorname{sech}(K \pi / 2 D)$ where $\lambda$ is an adjustable parameter. We show that such 'sech-weighted' differences include both the truncated sinc method and standard finite differences as special cases, corresponding to the limits $D \rightarrow \infty$ and $D \rightarrow 0$, respectively.

In the next to last section, we illustrate the use of sech-weighted differences in solving differential equation boundary value problems. The final section is a summary and prospectus. 


\section{The sinc pseudospectral method and truncation}

If the weights $\delta j^{(m)}$ are chosen so that (1.8) and (1.9) are the usual Fourier expansions of $K^{m}$ on $K \in[-\pi, \pi]$, that is,

$$
\begin{aligned}
& \delta_{j}^{(1)}=(-1)^{j+1} / j, \quad j=1,2, \ldots . \quad \text { (first derivative) } \\
& \delta_{0}^{(2)}=\pi^{2} / 3, \\
& \delta_{j}^{(2)}=2(-1)^{j} / j^{2}, \quad j=1,2, \ldots . \quad \text { (second derivative) }
\end{aligned}
$$

and so on, then (1.8) and (1.9) become, in the limit of infinite $n$, the usual sinc pseudospectral formulas. This is easily verified by comparison of (1.8) and (1.9) with the sinc differentiation formulas of $\left[1 ; 3\right.$, p. 722] or [4], noting that $D_{2}$ is proportional to the negative of the second derivative.

Because we have assumed $u(x)$ is exponentially decaying for large $|x|$, it follows that the derivative sum (1.5) will differ from the infinite series by an amount which decreases exponentially with $n$ for $n \gg 1$ since the contributions from $u(x+j h)$ for huge $|j|$ will be negligible. Thus, although the infinite series must always be truncated to finite $n$ for computational evaluation, we can effectively obtain the accuracy of the full infinite series (to within machine roundoff error) if $n$ is sufficiently large. Since the Fourier series for $K^{m}$ are convergent, it follows that in this 'pseudospectral limit' of $n \gg 1$, there is zero error in the differentiation formulas for all wavenumbers $|k|<k_{\text {limit }}$. Exact differentiation for all wavenumbers within the aliasing limit is in fact a generic property of spectral methods [3].

Unfortunately, achieving the pseudospectral limit may require summing hundreds of terms. Boyd [1] experimented with truncating the sinc derivative series to small and moderate $n$ where 'moderate' in this context means that the $u(x+j h)$ are of the same order of magnitude as $u(x)$ so that the decrease in the terms of the series has to come primarily from the decrease of the weights $\delta_{j}^{(m)}$ with $j$. Unfortunately, as shown in Fig. 1 of [1], the truncated sinc series converge with unacceptable slowness.

We can understand why by combining (2.1) with (1.8) and (1.9), the Fourier sine series for $K$ does
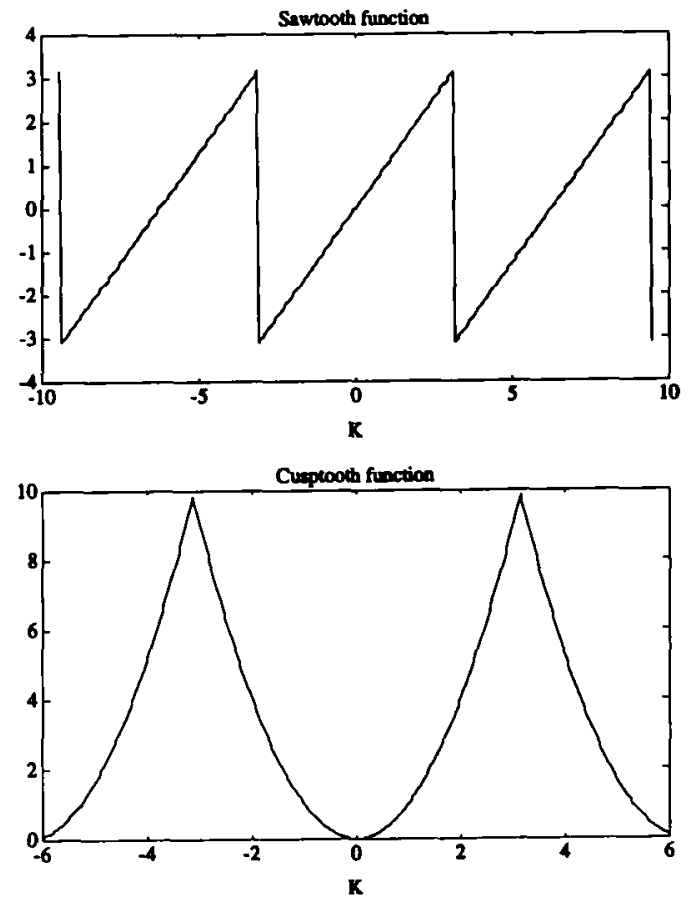

Fig. 1. (a). A graph of the 'sawtooth' function, which is defined as the sum of the Fourier series $f=2 \sum_{j-1}^{*}\left[(-1)^{i+1} / j\right] \sin (j K)$. (b) Same as (a) but for the 'cusptooth' function, which is the Fourier series for $K^{2}$, that is, the sum of $f=\left(\pi^{2} / 3\right)-4 \sum_{j=1}^{x}$ $\left[(-1)^{\prime+} / j^{2}\right] \cos (j K)$. 
not actually converge to $K$, but rather to the so-called piecewise-linear 'sawtooth function', which is equal to $K$ on the interval $K \in[-\pi, \pi]$ and is defined by periodicity for all other $K$ (Fig. 1(a)). Because of the jump discontinuities at $K= \pm \pi$, the Fourier coefficients decrease only as $\mathrm{O}(1 / j)$ and the sawtooth function is widely used in texts as the very paradigm of a slowly converging Fourier series.

The corresponding function for the second derivative, Fig. 1(b), is continuous with first derivative discontinuities; we may call it the 'cusptooth' function since it has cusps at $K= \pm \pi$. The coefficients decrease more rapidly as $\mathrm{O}\left(1 / j^{2}\right)$ because the function is continuous in $K$, but this is still unacceptably slow.

In general, the sinc pseudospectral sum for the $m$ th derivative has terms decreasing as $\mathrm{O}\left(1 / j^{m}\right)$ until $j$ is sufficiently large so that the exponential decay of $u(x)$ becomes important. Truncating the sinc pseudospectral sums to small or moderate $n$ is a horrible idea because of the huge loss of accuracy thus incurred.

Another perspective is to recall that a Fourier series is a least squares approximation to the function being represented, in our case, $K^{m}$, with equal weight over the whole interval of approximation. For approximating derivatives of a function $u(x)$, equal weighting is incorrect because the Fourier transform $U(k)$ will decrease exponentially with $k$ if $u(x)$ has the smoothness and decay properties we have assumed. It follows that our goal is not to differentiate all wavenumbers within the aliasing limit with equal accuracy. Rather, we want to do especially well for small wavenumbers (where $U(k)$ is large) and can cheerfully accept large errors near the aliasing limit where $U(k)$ is small. (If $U(k)$ is not small near the aliasing limit, then this implies that aliasing errors from wavenumbers just above the aliasing limit will be large.)

Ordinary finite differences have just the sort of bias towards small $k$ (or $K$ ) which is required as we explain next.

\section{Finite differences as the acceleration of the sinc pseudospectral method}

If we write the $n$th order sinc derivative series as

$$
S_{n}=\sum_{j=0}^{n} a_{j}
$$

where

$$
\begin{aligned}
& a_{0}=\delta_{0}^{(m)} u(x), \\
& a_{j}=\delta_{-j}^{(m)} u(x-j h)+\delta_{j}^{(m)} u(x+j h), \quad j=1, \ldots, n,
\end{aligned}
$$

where the $\delta_{j}^{(m)}$ are the sinc weights defined by $(2.1)$, then the $n$th order finite difference approximation is

$$
D_{m}^{f d}=\sum_{j=0}^{\infty} w_{n j} a_{j}
$$

where the finite difference acceleration weights for both first and second derivatives are

$$
\begin{aligned}
& w_{n 0} \equiv\left(6 / \pi^{2}\right)\left\{\sum_{j=1}^{n} 1 / j^{2}\right\}, \\
& w_{n j}=(n !)^{2} /[(n-j) !(n+j) !], j=1, \ldots, n .
\end{aligned}
$$

(different weights are needed for the third and higher derivatives). We could, of course, combine (3.4) with sinc weights (2.1) to write the finite difference approximation directly in the form of (1.5). However, it is more illuminating to show that finite difference methods are the result of applying sum acceleration, with a certain set of acceleration weights, to the standard sinc pseudospectral method.

In the language of sum acceleration theory, the weights (3.4) are triangular, positive, regular and 
Toeplitz. 'Triangular' means that the $n$th order accelerated approximation can be computed using only the terms of the series of degree $n$ or less. 'Positive' means that all the weights are of the same sign. 'Regular' means that if the acceleration is applied to any convergent series, then in the limit $n \Rightarrow \infty$, the accelerated series, which has the form of the right-hand side of (3.3), must also converge, and converge to the same sum as the unaccelerated series. For finite differences, which may alternatively be derived by differentiating the Lagrangian interpolating polynomial through $(2 n+1)$ points, regularity is proved by the following.

FERRAR'S THEOREM [5-7]. In the limit that the number of interpolation points tend to infinity, the Lagrangian interpolating polynomial converges to the same limit as the sinc series if the latter is convergent for a given function $u(x)$ and grid spacing $h$. Conversely, if the polynomial approximation is convergent, then the sinc series is summable via the method of de la Vallee Poussin to the same sum.

Lastly, the 'Toeplitz' property, true also of the Euler acceleration of [1], means that the weights satisfy certain additional technical requirements. The collective significance of these four jargon terms is that the finite difference sum acceleration defined by the weights (3.4) is extremely robust and well behaved. We omit the elementary derivation of (3.4) and the discussion of the Toeplitz property, etc., because finite differences are familiar, but these details are given in the technical report [8].

By using the standard asymptotic expansion for the gamma function, it is easy to show that

$$
\begin{aligned}
w_{n j}=\frac{(n !)^{2}}{(n-j) !(n+j) !} & \sim \exp \left(-\frac{j^{2}}{\left(n+\frac{1}{2}\right)}\right), j=0,1, \ldots, n \\
& \sim \exp \left(-\frac{j^{2}}{\left(n+\frac{1}{2}\right)}-\frac{j^{4}}{6\left(n+\frac{1}{2}\right)^{3}}\right) .
\end{aligned}
$$

Fig. 2 compares the lowest order and improved asymptotic approximations, (3.5) and (3.6), respectively, with the exact weights for $j>0$. The agreement is excellent. The graphs confirm that the weights decay as a Gaussian of $j$, but the graphs also show that the decay is faster than Gaussian for large $j$ as expressed (in part) by the correction term in (3.6). Remarkably, at high order, the weights for large $j$
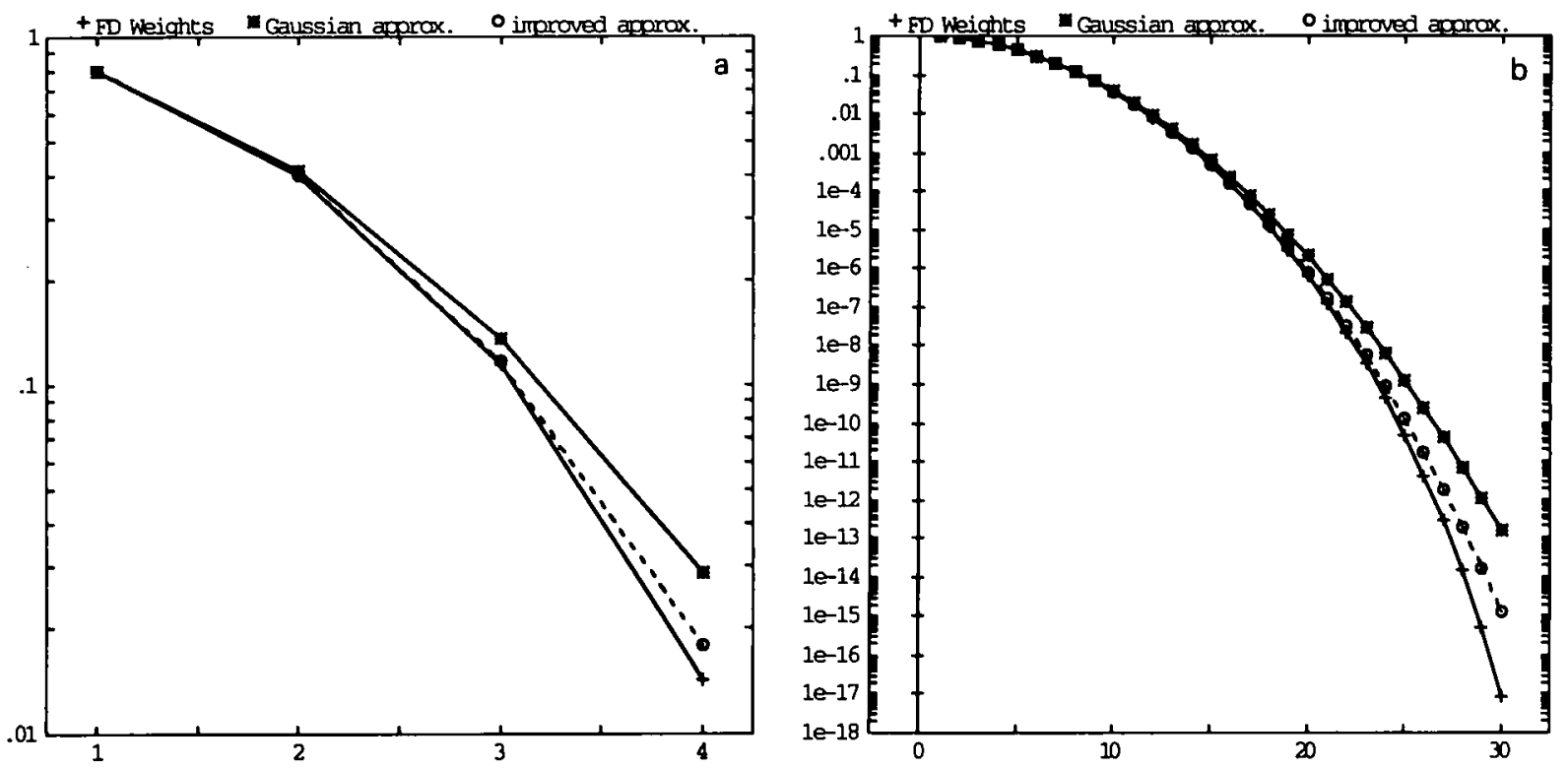

Fig. 2. (a). Bottom curve (crosses): the finite difference weights $w_{4 j}$ versus $j$. Middle curve (circles): the improved approximation (3.6). Top curve (asterisks): Gaussian approximation, $w_{n j} \sim \exp \left(-j^{2} /(n+1 / 2)\right)$. (b) Same as (a) for $n=30$ (60th order finite difference). 
are so small as to fall below the roundoff threshold; thus, one obtains $(2 n)$ th order accuracy to machine precision with fewer than $(2 n+1)$ points in the derivative series. Thus, if one neglects all terms with weights smaller than $10^{-10}$, say, then the $n=30$ (60th order) difference sum is truncated to span only 49 points.

Fornberg [9-11] has shown that the limit of high order finite differences is the pseudospectral method; it is interesting to see that the opposite is also true in the sense that the pseudospectral method can generate finite differences via sum acceleration. Nevertheless, one is still left with questions.

Are finite differences optimum? Or are there better alternatives? Can the truncated-sinc and finite difference methods be fitted in a common framework? In the next section, we give affirmative answers to both of the last two questions.

\section{Spectrally weighted least squares differences and the optimization theorem}

THEOREM (difference optimization). Define $\varepsilon_{m}(x)$ to be the error in the approximation of the mith derivative of $x$ by $a(2 n+1)$-point difference formula, i.e.

$$
\varepsilon_{m}(x) \equiv i^{-m} h^{m} \frac{\mathrm{d}^{m} u}{\mathrm{~d} x^{m}}-\sum_{j=-n}^{n} \delta_{j}^{(m)} u(x+j h) .
$$

For a given function $u(x)$, define the optimum weights $\delta_{j}^{(m)}$ to be those which minimize $\varepsilon_{m}(x)$ in the least squares sense:

$$
\Theta \equiv \int_{-x}^{\infty} \varepsilon_{m}^{2}(x) \mathrm{d} x
$$

Then the minimization problem can be expressed in wavenumber space by using the identity

$$
\Theta=\frac{1}{h} \int_{-x}^{\infty}\left|U\left(\frac{K}{h}\right)\right|^{2}\left|K^{m}-\sum_{i=-n}^{n} \delta_{i}^{(m)} \mathrm{e}^{i j k}\right|^{2} \mathrm{~d} K,
$$

where, as earlier, $K \equiv k h$.

In words, this means that the optimum weights for a given $u(x)$ are the spectral coefficients of the least squares expansion of $K^{m}$ with a weight function equal to the square of the Fourier transform of $u(x)$.

PROOF. Define the Fourier transform of $\varepsilon_{m}(x)$ as

$$
E_{m}(k) \equiv \frac{1}{\sqrt{2 \pi}} \int_{-x}^{x} \varepsilon_{m}(x) \exp (-\mathrm{i} k x) \mathrm{d} x
$$

Through elementary transform identities and the definition of $\varepsilon_{m}(x)$,

$$
E_{m}(k) \equiv\left\{k^{m} h^{m}-\sum_{j=-n}^{n} \delta_{j}^{(m)} \exp (\mathrm{ij} k h)\right\} U(k),
$$

where $U(k)$ is the Fourier transform of $u(x)$, defined as in (4.4). The Parseval Formula [12] implies

$$
\int_{-x}^{x} \varepsilon_{m}^{2}(x) \mathrm{d} x=\int_{-x}^{\infty}\left|E_{m}(x)\right|^{2} \mathrm{~d} x .
$$

But the left and right sides of (4.6) are merely the two equivalent definitions of $\Theta$, the function whose minimum defines the optimum weights.

The flaw in the theorem is that we normally do not know $U(k)$ in advance, and if we did, we would not need difference formulas for the derivatives since these can be computed directly by taking the inverse Fourier transform of $(i k)^{m} U(k)$. Nevertheless, we do know that for a smooth function, $U(k)$ should decay exponentially with $k$ for sufficiently large $|k|$. This knowledge of the qualitative form of $U(k)$ suggests the following generalized differences. 
DEFINITION (spectrally weighted least squares differences). SWLS differences are a derivative approximation of the form

$$
D_{m}^{\mathrm{app}} u(x) \equiv \sum_{j=-n}^{n} \delta_{j}^{(m)} u(x+j h),
$$

where the weights are chosen so the truncated Fourier series with coefficients $\delta_{j}^{(m)}$ is the usual least squares approximation to $K^{m}$ with the weight function $\omega(K)$, that is, the $\delta_{j}^{(m)}$ are chosen to minimize the integral $I$ defined by

$$
I=\int_{-\pi}^{\pi} \omega(K)\left(K^{m}-\sum_{j=-n}^{n} \delta_{j}^{(m)} \mathrm{e}^{\mathrm{j} j k}\right)^{2} \mathrm{~d} K
$$

If we define the inner product, for two arbitrary functions $f(K)$ and $g(K)$, as

$$
(f, g) \equiv \int_{-\pi}^{\pi} f(K) g(K) \omega(K) \mathrm{d} K
$$

let

$$
\varphi_{j}(K)= \begin{cases}\sin (j K), & m \text { odd } \\ \cos (j K), & m \text { even }\end{cases}
$$

and define the vectors and square matrix

$$
\left.\boldsymbol{\delta}\right|_{j}=\delta_{j}^{(m)},\left.\quad \boldsymbol{x}\right|_{j}=\left(K^{m}, \varphi_{j}\right),\left.\quad \boldsymbol{G}\right|_{i j}=\left(\varphi_{i}, \varphi_{j}\right), \quad i, j=0, \ldots, n .
$$

Then weights of the spectrally weighted least squares differences are computed by solving the matrix equation

$$
\boldsymbol{G} \boldsymbol{\delta}=\boldsymbol{\chi}
$$

The Gram matrix $G$ is poorly conditioned, so we solved it via the singular value decomposition (SVD) [13]. The definition of SWLS differences employs an integral truncated to the interval $K \in[-\pi, \pi]$, that is, to wavenumbers within the aliasing limit, for computational convenience. However, unless the $U(K / h)$ decays sufficiently fast that it is negligible for all wavenumbers outside the aliasing limit, then aliasing error will destroy the accuracy of the difference approximation. Consequently, the distinction between the infinite integration limits of the theory and the finite limits of (4.7) is meaningless unless the differences themselves are inaccurate.

The truncation of the sinc pseudospectral method is the special case of SWLS differences:

$$
\omega \equiv 1 \quad \text { (truncated-sinc) . }
$$

Finite differences correspond to choosing the weights $\delta_{j}^{(m)}$ so that the Taylor expansion of the truncated Fourier series agrees with $K^{m}$ to as high a degree as possible. Since all the stress is on emphasising accuracy in the limit $K \rightarrow 0$,

$$
\omega(K)=\delta(K) \quad \text { (finite difference), }
$$

where $\delta$ is the Dirac delta function [12].

The Fourier transform of a typical $u(x)$ is neither infinitely concentrated at the origin nor evenly distributed over all wavenumbers inside the aliasing limit. Rather, $U(k)$ resembles the hyperbolic secant function. This suggests trying 'sech-weighted' differences,

$$
\omega(K) \equiv \operatorname{sech}(K \pi /(2 D)) \quad \text { (sech-weighted) , }
$$

where $D$ is an adjustable constant.

The drawback of sech-weighted differences is that one usually does not know a priori how fast $U(k)$ will decay. However, one usually does have a desired error tolerance $\mu(\ll 1)$ in mind. If the Fourier transform of $u(x)$ does not decay sufficiently fast so that $U\left(k_{\text {limit }}\right) \leqslant \mu$, then the calculation will fail 
(smaller grid spacing $h$ is needed). It follows that for a successful calculation, unspoiled by aliasing error, we can put an upper bound on $D$. Using this bound

$$
D=-\pi^{2} /(4 \log (\mu))
$$

will give a weight function of finite width and should thus improve upon finite differences.

Of course, if $U\left(k_{\text {limit }}\right)$ is very small in comparison to $\mu$, then (4.15) is not optimum because a narrower weight function (smaller $D$ ) would better fit the fast decaying $U(k)$. However, this only happens when we have chosen a grid spacing $h$ which is smaller than needed to achieve the desired error tolerance $\mu$. With such a fine grid spacing, a non-optimum choice of $D$ will not prevent the difference formulas from being accurate to within the desired tolerance. Consequently, (4.15) is a safe choice for $D(\mu)$ whenever the grid spacing is small enough so that an error less than $\mu$ can be achieved.

\section{Numerical examples}

To test different algorithms, we solved two differential equations, both with a grid spacing $h=3 / 10$ and a grid of 201 evenly spaced points spanning the interval $x \in[-30,30]$. The solutions decay exponentially so that the error in approximating the infinite interval by a finite interval is negligible. The differential equations are

$$
\begin{array}{ll}
u_{x x}-u=-2 \operatorname{sech}^{3}(x), \quad u(x)=\operatorname{sech}(x) & \text { (Case I) } \\
u(x)=\operatorname{sech}(x) \cos \left(\left[\frac{\pi}{2 h}\right] x\right) \quad \text { (Case II) } \\
u_{x x}-u=\left\{-2 \operatorname{sech}^{3}(x)-\frac{\pi^{2}}{4 h^{2}} \operatorname{sech}(x)\right\} \cos \left(\left[\frac{\pi}{2 h}\right] x\right)+\frac{\pi}{h} \operatorname{sech}(x) \tanh (x) \sin \left(\left[\frac{\pi}{2 h}\right] x\right) .
\end{array}
$$

Case $\mathrm{I}$ is chosen so that its solution has a Fourier spectrum $U(k)$ which is concentrated near $k=0$, as true of most randomly selected functions. Case Il was chosen so that the $\cos ([\pi / 2 h] x)$ factor would produce a spectrum with peaks at wavenumber $k= \pm k_{\text {limit }} / 2$ where $k_{\text {limit }}(=\pi / h)$ is the aliasing limit for the given grid. This Fourier spectrum is typical of a wave packet where $\operatorname{sech}(x)$ is the 'modulation' or 'envelope' and the rapidly varying oscillatory factor $\cos \left(0.5 k_{\text {limit }} x\right)$ is the 'carrier wave'.

In making comparisons, we used a number of alternative algorithms besides those described earlier. The 'truncated-sinc' method of [1] simply chops off the usual pseudospectral derivative sums to include only $(2 n+1)$ terms centered on the point where the derivative is to be evaluated. The 'Euleraccelerated sinc' not only truncates the sums, but multiplies them by the weights of the Euler acceleration method as described in [1]. The 'Padé-accelerated sinc' multiplies the terms of the truncated-sinc series by $z^{\prime}$, forms Padé approximants in $z$ as described in [14], and then evaluates the result at $z=1$. The 'Levin $u$-transform' is a non-linear acceleration scheme which was found by Smith and Ford to be the best general purpose acceleration [15-17].

Fig. 3(a) shows that the truncated-sinc method, as expected, is terrible. The sech-weighted difference scheme with $D=0.15$ is best followed by finite differences and the Euler acceleration. The difference between the sech-weighted differences and standard finite differences is only little more than a factor of 2 for most $n$, but this still confirms the analysis in Section 4 .

The Fourier transform for $\operatorname{sech}(x)$ is

$$
U^{2}(K / h)=\frac{\pi}{2} \operatorname{sech}^{2}\left(\frac{\pi}{2} \frac{K}{h}\right) \sim \pi \exp \left(\frac{\pi}{2} \frac{K}{h / 2}\right), \quad K \gg h,
$$

which shows that analytically, the optimum width parameter in the sech-weighting for $u=\operatorname{sech}(x)$ is $D=h / 2$, as used in Fig. 3(a).

If we choose the error tolerance $\mu$ to equal $U\left(k_{\text {limit }}\right)$, which is the smallest the error can be for a given choice of grid spacing $h$, then (4.15) also suggests $D=h / 2$.

Fig. 3(b) compares the truncated-sinc and finite difference schemes, already shown in Fig. 3(a), with 

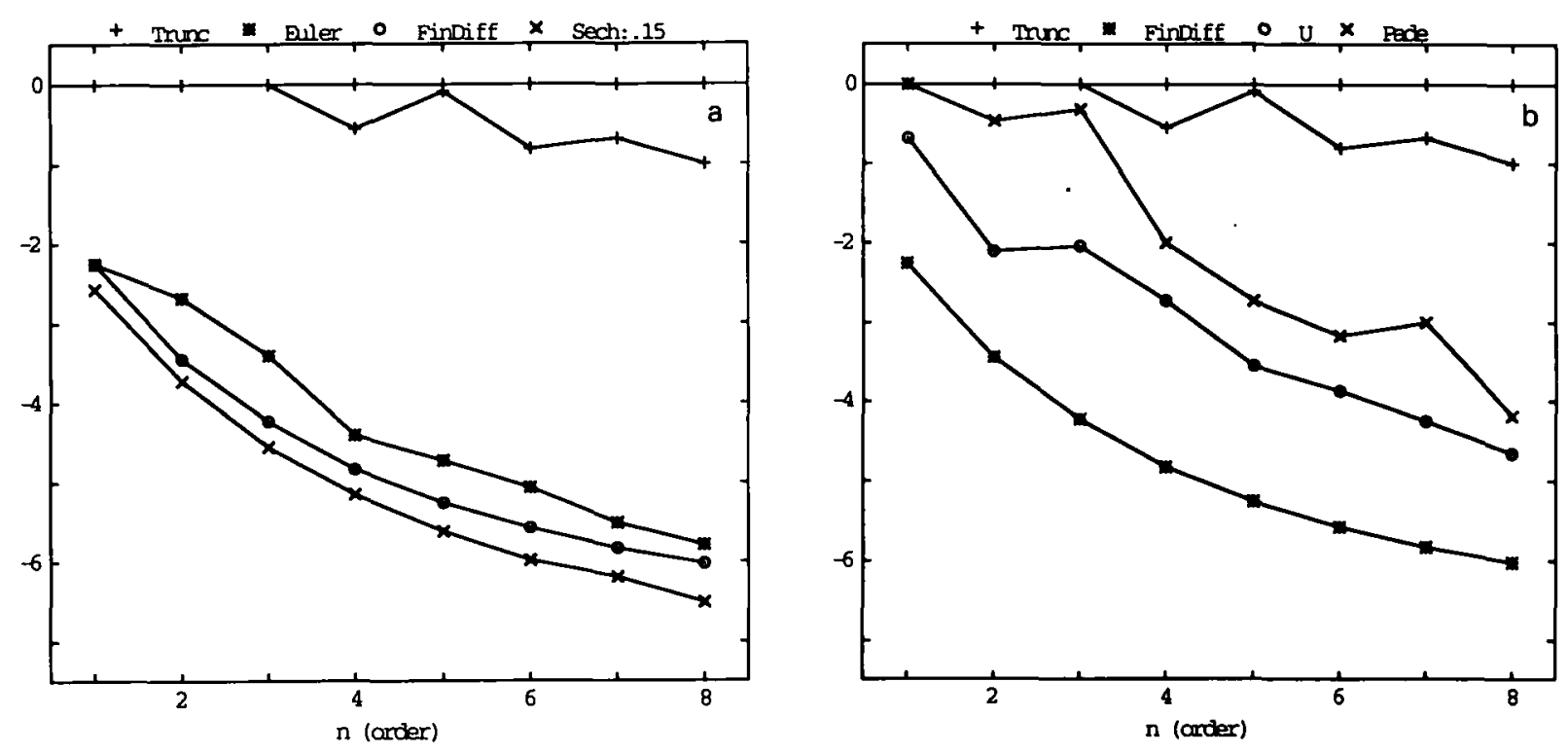

Fig. 3. (a). Solutions to Case I, exact solution $u=\operatorname{sech}(x)$, using various methods. Top curve (plus signs): truncated sinc. Second from top (asterisks): Euler-accelerated sinc. Second from bottom (circles): finite difference. Bottom (crosses): sech-weighted differences, $D=0.15$. (b) same as (a) except two of the four methods are different. Top curve (plus signs): truncated sinc. Second from top (crosses): Padé-sinc. Second from bottom (circles): Levin $u$-transform. Bottom (asterisks): finite difference.

the two non-linear accelerations. It is clear that while both Padé approximants and the Levin $u$-transform are better than truncation, both are much worse than finite differences or indeed any of the three linear accelerations shown in Fig. 3(a).

The reasons for the failure of the non-linear schemes, so highly praised in the sum acceleration literature [15-18], are discussed in [2], but the key issue is uniformity. An effective derivative approximation must be accurate for a broad range of wavenumbers. The $u$-transform and Padé schemes are too non-uniform in wavenumber to be effective.

Fig. 4(a) shows that for Case II, the advantages of sech-weighted differences (with $D=1 / 4$ ) over finite differences are much more pronounced: roughly a factor of 10 . The Fourier transform of the wave-packet-like function $u(x)$ is peaked at $k= \pm k_{\text {limit }} / 2$, so both the $\delta$-function weighting of finite differences and the sech-weighting, which decay from peaks at $k=0$, are very different in shape from $U^{2}(K / h)$. However, both accelerations converge anyway. Because of the finite width of its weighting function, the sech-weighted differences are much more effective at differentiating the higher wavenumbers than finite differences.

Fig. 4(b) shows that even the lowly and ancient Euler acceleration is better than finite differences for almost all $n$. (Although not shown in Fig. 4(a), the Euler method is better than finite differences for Case I, too, but only for $n>10$.). Because the Fourier spectrum of $u(x)$ is broad, the truncated-sinc method compares much more favorably with the accelerated schemes than was true for Case $I$.

\section{Summary}

The optimum difference scheme for approximating the $m$ th derivative of a function $u(x)$ is one whose weights are chosen to be those of a least-squares approximation of $K^{m}$ by a Fourier series in wavenumber with a weight function equal to the square of $U(K / h)$ where $U(k)$ is the Fourier transform transform of $u(x)$. In practical applications, $U(k)$ is not known a priori. Nevertheless, it is possible to exploit knowledge about the qualitative shape of $U(k)$ to produce a more effective scheme than classical finite differences. Our numerical examples show that 'sech-weighted' differences work very well for problems whose solution has a Fourier transform that decays geometrically with wavenumber.

We also experimented with a wide variety of non-linear accelerations, that is, acceleration methods 

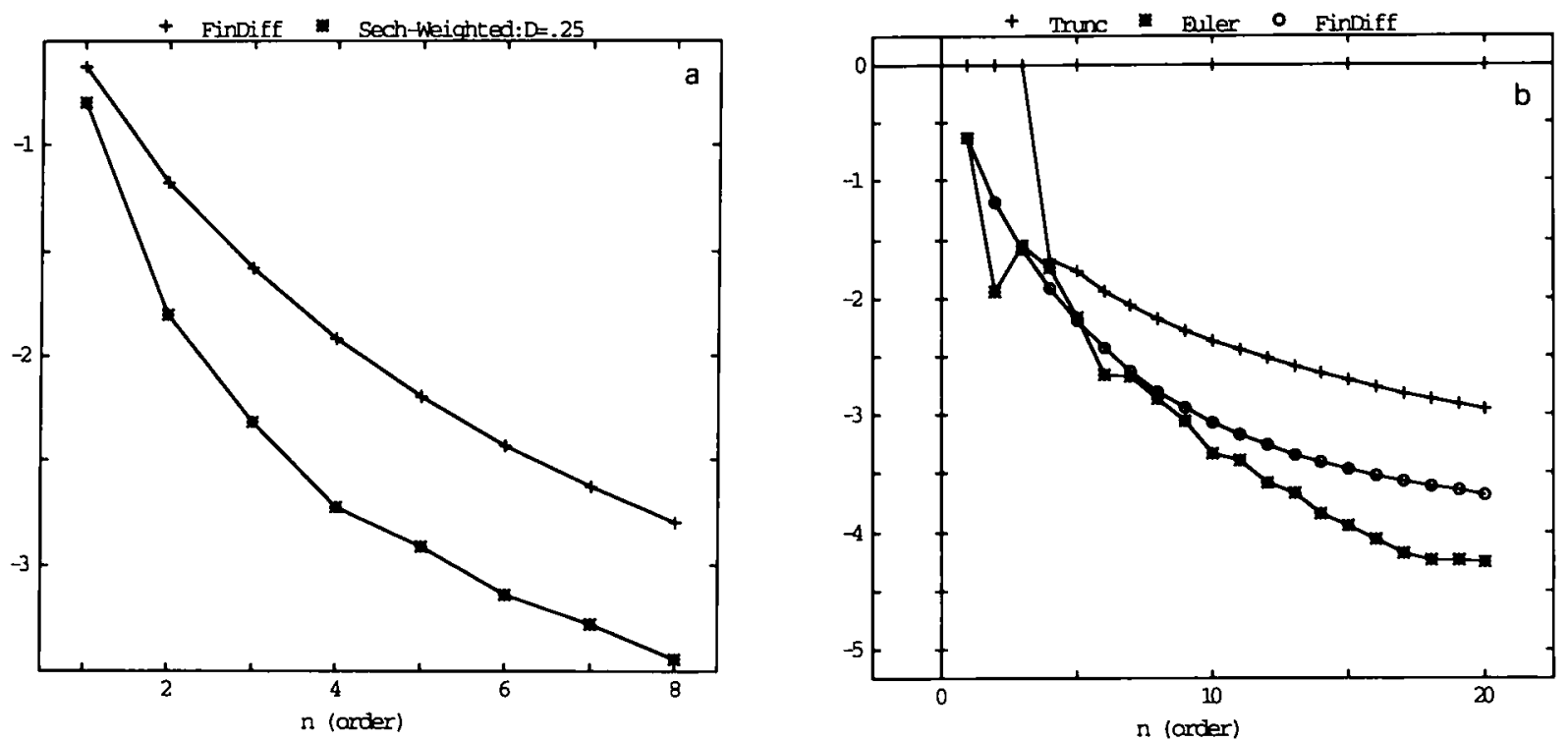

Fig. 4. (a). Solutions to Case II where $u(x)=\operatorname{sech}(x) \cos \left(\left(k_{\text {limit }} / 2\right) x\right)$ where $k_{\text {lumit }}=\pi / h$ is the aliasing limit. Top curve (plus signs): finite difference. Bottom curve (asterisks): sech-weighted differences with $D=1 / 4$. (b) Same as (a) except for different methods. Top curve (plus signs): truncated-sinc. Middle curve (circles): finite difference. Bottom curve (asterisks): Euleraccelerated sinc.

for which the answer is a non-linear function of the terms of the original pseudospectral series. Padé approximants and the Levin $u$-transform both were much inferior to all the linear accelerations we tried. Both are probably better than the alternatives for small wavenumber [2], but their extreme non-uniformity in $k$ generates much larger errors for the spectrum as a whole.

Turbulent flows have amplitudes which decay as $k^{-1 / 2}$ (two dimensions) or as $k^{-5 / 6}$ (three dimensions). It follows that the Fourier spectra for turbulent flows are very broad, and finite differences particularly inappropriate. It seems likely that spectrally weighted least squares differences, using a weighting $w(K)$ which decays algebraically rather than exponentially with $K$, would be greatly superior to standard finite differences. (However, P. Moin has noted in his address at ICOSAHAM '92 that wavenumbers near the aliasing limit may be so corrupted by non-linear aliasing that accurate differentiation of these components is not important). A test of algebraically weighted differences in turbulence modelling is left for the future.

\section{Acknowledgment}

This work and $[19,20]$ were the basis for an invited plenary address at ICOSAHOM 92 in Montpellier, France. I wish to thank the organizers, Christine Bernardi and Yvon Maday, and the members of the Scientific Committee for the invitation to speak and for their hard work in orchestrating the conference. I also wish to thank SPIA and the other sponsors of ICOSAHOM. Lastly, I am appreciative to the National Science Foundation and the Department of Energy which supported this work through grants OCE8812300, DMS 8716766, ECS9012263, OCE9119459 and KC070101.

\section{References}

[1] J.P. Boyd, Sum-accelerated pseudospectral methods: the Euler-accelerated sinc algorithm, Appl. Numer. Math. 7 (1991) 287-296.

[2] J.P. Boyd, A fast algorithm for Chebyshev and Fourier interpolation onto an irregular grid, J. Comp. Phys. 103 (1992) 243-257. 
[3] J.P. Boyd, Chebyshev and Fourier Spectral Methods, Lecture Notes in Engineering 49 (Springer, New York, 1989).

[4] J. Lund and K.L. Bowers, Sinc Methods for Quadrature and Differential Equations (SIAM, Philadelphia, 1992).

[5] J.M. Whittaker, Interpolatory Function Theory (Stechert-Hafner, New York, 1964) 64.

[6] W. Ferrar, Proc. Roy. Soc. Edinburgh 45 (1925) 269.

[7] W. Ferrar, Proc. Roy. Soc. Edinburgh 46 (1925) 323.

[8] J.P. Boyd, Summation methods and pseudospectral algorithms, Part I: Finite difference summation of Whittaker cardinal (sinc) series, Technical Report, Laboratory for Scientific Computation, University of Michigan, 1987, $50 \mathrm{pp}$.

[9] B. Fornberg. On a Fourier method for the integration of hyperbolic equations, SIAM J. Numer. Anal. 12 (1975) $509-528$.

[10] B. Fornberg. The pseudospectral method: comparisons with finite differences for the elastic wave equation, Geophysics 52 (1987) 483-501.

[11] B. Fornberg, High-order finite differences and the pseudospectral method on staggered grids, SIAM J. Numer. Anal. 27 (1990) 904-918.

[12] G.F. Carrier, M. Krook and C.E. Pearson, Functions of a Complex Variable (McGraw-Hill, New York, 1966).

[13] W.H. Press, B.P. Flannery, S.A. Teukolsky and W.T. Vetterling, Numerical Recipes: The Art of Scientific Computation (Cambridge University Press, New York, 1986).

[14] C.M. Bender and S.A. Orszag, Advanced Mathematical Methods for Scientists and Engineers (McGraw-Hill, New York, 1978).

[15] D.A. Smith and W.F. Ford, Acceleration of linear and logarithmic convergence, SIAM J. Numer. Anal. 16 (1979) 223-240.

[16] D.A. Smith and W.F. Ford, Numerical comparisons of nonlinear convergence accelerators, Math. Comp. 38 (1982) $481-499$.

[17] E.J. Weniger, Nonlinear sequence transformations for the acceleration of convergence and the summation of divergent series, Comput. Phys. 10 (1989) 189-371.

[18] E.J. Weniger, On the derivation of iterated sequence transformations for the acceleration of convergence and the summation of divergent series. Comput. Phys. Comm. 64 (1991) 19-45.

[19] J.P. Boyd, Defeating the Runge phonomenon for equispaced polynomial interpolation via Tikhonov regularization. Appl. Math. Lett. 5 (1992) 57-59.

[20] J.P. Boyd, A lag-averaged generalization of Euler's method for accelerating series, Appl. Math. Comput. (1992) submitted. 